\title{
Quelques monuments byzantins inédits.
}

(Amulettes, méreaux, etc.)

\section{1}

Amulette en forme de médaille uniface, de cuivre youge, oravée en creux, de 52 millim. de diamètre environ, percée d'ux trô de suspension. Cette amulette était destinée à être portée ay col - Cabinet des Médailles de France. - Voici la description de conument:

Le Christ au nimbe crucigère, vêtu d'ung longue robe jusqu'aux pieds, est fixé sur la croix. Ses pieds touchent presque à terre tant la croix est peu élevée. A ses côtés les doly larrons, vêtus d'un simple jupon, également crucifiés sur une crohr/très courte. On aperçoit les liens qui attachent leurs pieds. Kes seins sont indiqués pour bien marquer qu'à l'encontre du Chyist los larrons ont le buste nu. Les deux croix latérales sont terpinnées en forme de tau par. une tablette destinée au titre. Le troư de syspension pratiqué après coup, précisément au dessus du njúbe du/Christ, ne permet pas de constater si la croix médiane prégentait la même disposition. Les bras des trois crucifiés sont si mal indiqué qu'ils semblent se confondre avec les extrémités mêmes de la baye transversale des croix. - Aux pieds du Christ deux adorants sont accroupis qui lui présentent chacun un objet difficilement reconnaissoble; l'un de ces objets semble un verre à boire. Quels sont ces deux personnages? Assurément ni la Vierge et Saint Jean ni les deux sqldats constamment représentés debout l'un avec la lance, l'autre avec/'éponge imprégnée de vinaigre.

A droite et a gauche de la tête du Christ les lettres de son nom EMMANชH^ $\mathrm{Au}$ dessous de la scène du crucifiement l'inscription

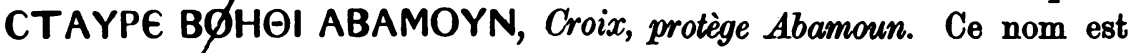
certainement celui du propriétaire de l'amulette.

$\mathrm{Au}$ desssous encore la scène des saintes femmes au tombeau occupe toute la/partie inférieure du champ. A gauche, les saintes Marie et Marthe nimbées, désignées par leurs noms MAPIA S (pour KAI) MAPOA, s'avancent, la main au visage, dans l'attitude de la douleur. 
Celle qui marche en avant porte une fiole (?) à parfum à goulot trés allongé. A droite l'ange assis sur un siège, s'adressant fux femmes,

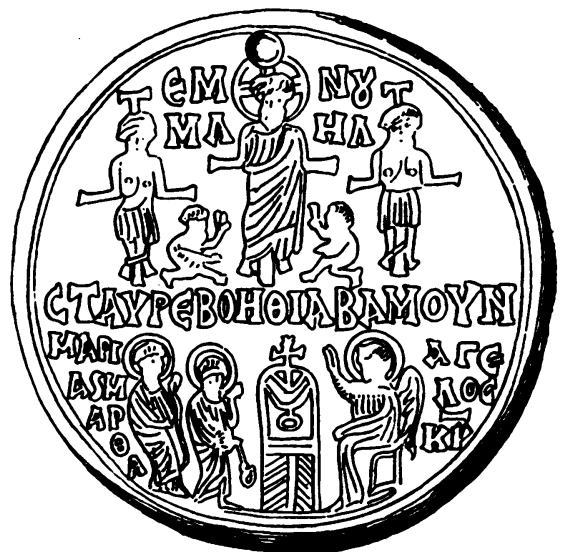
lève la main droite. Perrière lui son nom AГE^OC sic) $\overline{K Y}$ pour ATГE^OC KY FIOY, l'Ange du Seigneur. Au ceptre, le tombeau surmonté d'une coix, à façade ornementée, avec/porte à deux battants.

Tous los détails de ce très curieux nonument, la forme des croix, le Christ représenté vêtu, etc., sont cgractéristiques d'une époque ancie ne et d'une origine orientale. Le lom du propriétaire se retrouve day Jamblique sous la forme ( $\left.\beta \dot{\alpha} \mu \mu \omega \nu .{ }^{1}\right)$

Je pense qu'on peut considére cette amuletté comme provenant d'Egypte et comme ayant été fabpquée vers tê $\mathrm{VI}^{\mathrm{me}}$ ou VII'me siècle.

Amulette contre les maux A'estomac, en forme de médaille, formée d'une matière cornée brune, d 25 millim. de diamètre environ, percée d'un trou de suspension poun être portée au col.

$A u$ droit: Le sacifice A'Issac. Au centre Abraham tenant de la main gauche Isaag placé of sa droite, levant la droite pour le frapper

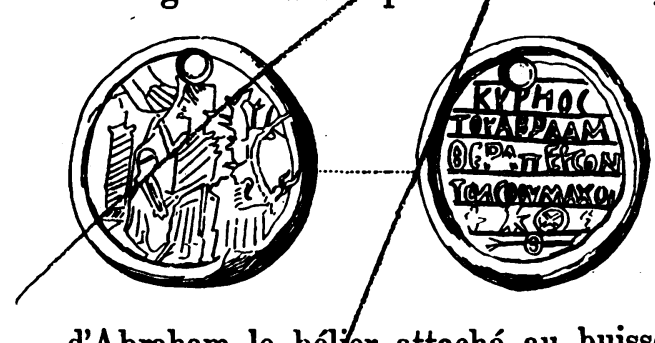
du couteau. Derrière Isaac l'autel en forme de fat de colonne sur une base. Au dessus de l'autel Dieu sous la forme d'une dextre divine issant des nuages représentés par une simple ligne en demi-cercle. A la gauche d'Abraham le bélier attaché au buisson. - Cette scène est représentée d'une manière toyes rudimentaire.

1) Voyez Hape-Benseler: Wörterbuch der griechischen Eigennamen. - 'A $\beta \alpha \dot{\alpha} \mu$ -

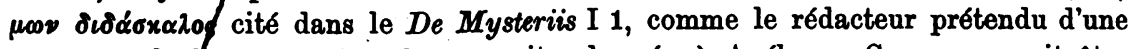
réponse à la ettre que Porphyre avait adressée à Anébo. «Ce nom parait être une transcriftion de la forme respectueuse $a b b a$, $a m b a$ Ammon d'un individu s'appelant Ammon (cf. Zœga, Cat. 130, 18. 38). Les individus portant le nom d'Ammon pont fréquents vers les Ve ou VIme siècles de notre ère». - Note communiquée/par M. Maspéro. 
Au revers: Légende en quatre lignes séparées par de traits: KYPHOC TOY ABPAAM OEPATTEYCON TON COWMAXON (sic), Seigneur d'Abraham, guéris (mon) estomac. Au dossous deux lignes de signes cabalistiques. Le tout dans un cercle. Cette curieuse amulette d'origine également orientale, d'époque chrétienne ancienne, est entre les mains d'un marchand d'antiquités de Pays.

\section{3}

Méreau de cuivre jaune ayant servi aux auhônes de la diaconie du célèbre couvent de $S^{t}$ Jean Baptiste de Stoydion, ou vulgairement de Stoudion tout court, à Constantinople. $\mathrm{XI}^{\mathrm{me}}-\mathrm{XII}^{\mathrm{me}}$ siècle.

Au droit: Entre les sigles accoutumes $\overline{\mathrm{MP}} \overline{\Theta \varnothing}$, la Vierge mi-corps, tournée à gauche, les mains dons l'attitude de l'oraisorr, la

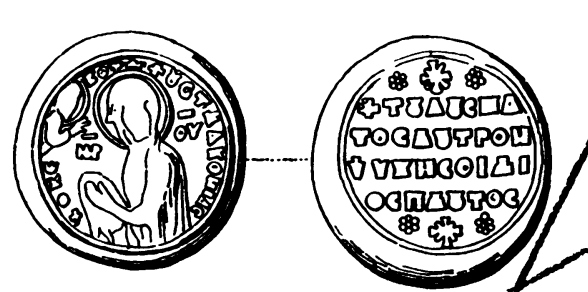

tête inclinée devant le piré éternel vy en buste dans les nuées, qui 1 bénit. Elle semble tenir de ses deur mains un objet qui poupont bien être son voile, le fameux maphorion. La légende de ce côté est: + $\Theta K \in B^{\prime} \odot^{\prime} T^{\prime} \Delta^{\prime}$

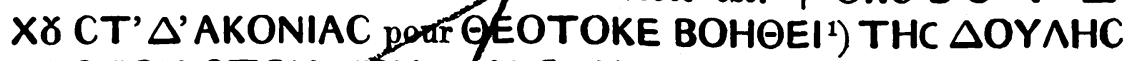
XPICTOY CTOY $\triangle$ TOY $\triangle A$ AKONIAC, Théotokos, protège la diaconie de Stoudion, serounte du Chfist.

Au yecers: Entre deax petites croix recroisetées cantonnées de rosettes la légende en quatre lignes: + T૪ $\ \varnothing C M A T O C \wedge \gamma T P O N$

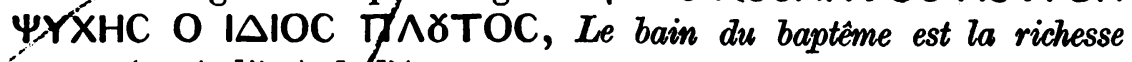
propre (particulière) de l'âme.

Diaconie doit arpir ici le sens de Service de la charité, des aumônes. La diaconie de Stoufion devait être le Service des aumônes de ce grand couvent. Le mérehu que je décris devait être employé à ce service. ${ }^{2}$ ) Dans ma Sigillog/aphie byzantine, à la page 139 , j'ai publié le sceau d'un higoumène de ce monastère, un des plus célèbres de Byzance, plus un sceau du courent même portant la représentation du baptême du Christ. Dans/la seconde série de Sceaux byzantins inédits publiée par moi dans la Revue des études grecques de 1891 , j'ai décrit sous le $\mathrm{n}^{\circ} 36$ le sceau d'un autre higoumène du Stoudion. Dans cette même seconde série j'ai fecrit sous le $\mathrm{n}^{\circ} 35$ un autre sceau de diaconie.

1) Ic par exception BOHOEl régit le génitif.

2) Sur l'usage de ces méreaux à Byzance voyez mon mémoire de la Revue archéologique de $188 \mathrm{C}$ (oct.) insitulé: Monumenis numismatiques et sphragistiques du mosen-âge byzantin. 
Méreau de cuivre jaune ayant servi aux aumônes du sébagtophore Jean Pépagoménos. - Cabinet de France - $\mathrm{XI}^{\mathrm{me}}-\mathrm{X} \mathrm{XI}^{\mathrm{me}}$ sjecle.

$A u$ droit: L'inscription en cinq lignes: + CY XE (pou/XPICTE)

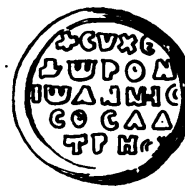
$\triangle W P O N$ IWANNHC COC . $\triangle A$ TPHC.

Au revers: Suite en cinq lignes de la même inscription: CEBA CTOФOPOC TTETTATOMENQC ФEPEI.

Cette inscription qui forme deux trimètres iambiques signifie je pense: Toi, Christ, le sebastophore Jean Pépagogénos ton adorateur (te?) présente (offre) ce don.

A la page 689 de ma Sigillographie byzantine j'ai décrit le sceau d'un Théodore Pépagoménos. A la page 85 j'ai décrit celui d'un sébastophore et cité un autre. - Un Démetrius Pépagoménos a écrit un traité sur l'art de soigner les faucons. ${ }^{1}$ ) Un autre. Pếpagoménos figure parmi les correspondants do Théodore Hyrtákénos au commencement du XIV ${ }^{\mathrm{me}}$ siècle. ${ }^{2}$ )

Bague en or du Musée yiqué à Venise (ancien Musée Correr). Cette bague, citée sous le 956 du Catalogue du Musée Correr par V. Lazari; Venise 1859/ est à huit pans. Sur l'un d'eux est fixé le

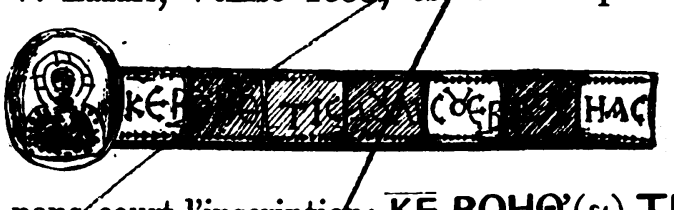
chaton de forme ovale où figure un Christ nimbé émaillé de travail assez grossier. Sur les sept autres

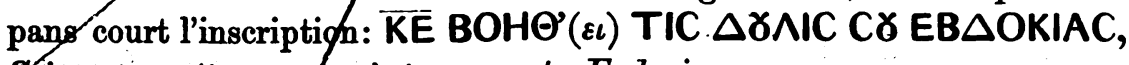
Seigneur, prête secoyfs à ta servante Eudoxie.

Cette bague au poids de 10 gr. 58 a certainement appartenu à quelque femme de haute naissance. L'émail était à Byzance chose fort précieuse. Ce bijou pourrait bien avoir été la propriété de quelqu'une des Eudoxie gui furent impératrices au IX ${ }^{\text {ne }}$ ou au $\mathrm{X}^{\text {me }}$ siècle.

\section{6}

Bagye en or de ma collection provenant de Trébizonde. Bague à huit pans du poids de 12 gr. Sur le chaton de forme ovale qui recouve un de ces pans est figurée la scène de l'Annonciation. La

1) Krumbacher, Gesch. der byzant. Litter., p. 68.

2) Ibid., p. 201. 
G. Schlumberger: Quelques monuments byzantins inédits

Vierge devant l'Ange file la laine qui sort de la corbeille traditighnelle. Les deux personnages étaient certainement émaillés primitivemght, mais l'émail a disparu. Le travail de la gravure est ici aussi asse grossier.

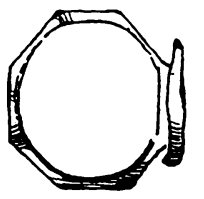

$$
\begin{array}{|l|l|l|l|l|l|l|}
\hline \theta \in O & T O K & E B H & \theta I T: N A^{8} & \triangle I N(T A G P \\
\hline
\end{array}
$$

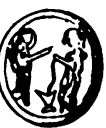

Sur les sept autres pans court l'inscription en caractéres très dé-

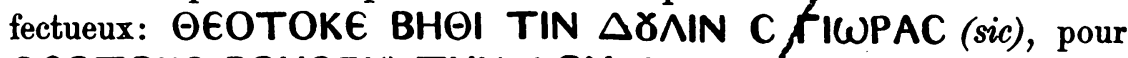

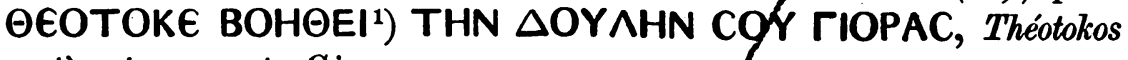
protège ta servante Gioras.

Le nom de la propriétaire de ce bijoy n'est point grec; ce doit être quelque nom ibère, géorgien ou arméfien.

Plaque circulaire et bombée de bronze, de 36 minillim. de diamètre, qui devait constituer une des deux portions d'une boîte ou capsule, sorte de phylactère ou d'enkolpion destiné à être porté au col. Musée civique de Venise ${ }^{2}$. Epoque des Paléologues.

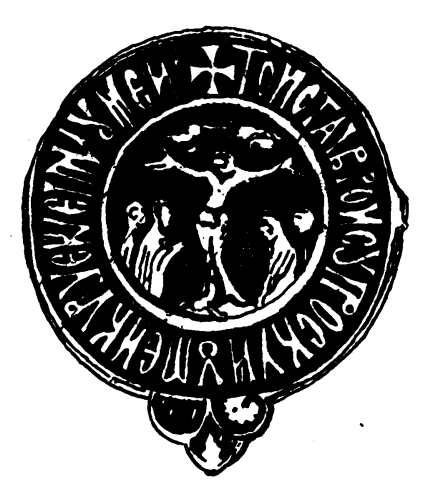

Sur la face concave est figuré le repas d'Emmaüs. - Sur la face convexe on voit/la Crucifixion. Quatre personnages sont agenouillés au pied de la croix. Deux chérubins l'accostent dans les airs. L'in-

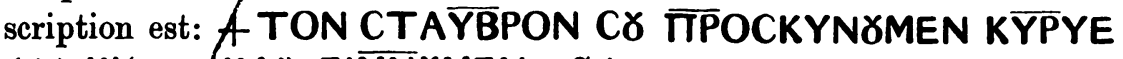
(sic) K'(pour KAI) EIMNXMEN, Seigneur, nous adorons et chantons ta croix.

Paris, 31 Mars 1893.

Gustave Schlumberger.

1) C: eOHEEI régit l'accusatif.

2) No 996 du Catalogue du Musée Correr de V. Lazari.

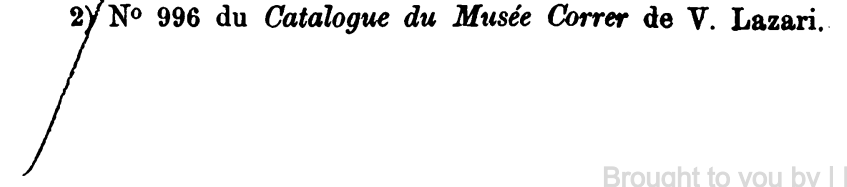

